\title{
Kinetic estimation of the adsorbate distribution on the surface from adsorbed amounts
}

\author{
Mehmet Polat* \\ Department of Chemical Engineering, Izmir Institute of Technology, Gulbahce, Urla 35430, Izmir, Turkey
}

Received 23 November 2005; accepted 27 December 2005

Available online 30 January 2006

\begin{abstract}
A phenomenological multilayer adsorption model for a well-dispersed, homogeneous, nonporous adsorbent and a molecular adsorbate is presented. The model provides explicit kinetic expressions associating the adsorbed amounts to the fraction of the surface occupied and reduces to the first- and second-order adsorption models for special cases. Parameters of the model are a pair of true rate constants related to the adsorbateadsorbent and adsorbate-surface adsorbate affinities. A general graphical procedure and analytical equations for special cases are provided to estimate the rate constants from kinetic adsorption data. Data from the adsorption of sodium stearate onto $\alpha$-alumina from water were used to test the model. The predicted values of the rate constants suggested that the stearate was distributed homogeneously on the alumina surface and essentially adsorbed as a monolayer before starting to form the second layer.
\end{abstract}

(c) 2006 Elsevier Inc. All rights reserved.

Keywords: Adsorption; Modeling; Kinetics; Multilayer

\section{Introduction}

Modeling the kinetics of adsorption is important for simulation, scaling-up, and control purposes. The film-pore model [1], the branched-pore diffusion model [2], and the film-solid model [3] are significant attempts that originate from first principles, but their solutions are numerical in nature. Phenomenological models where the various parameters of the system are lumped into empirical constants are another approach to analyzing the adsorption data. The first-order model [4] is the classical example and has been employed in numerous systems [5-23]. The rate equation and its solution in terms of fractional adsorbate concentration in solution are in the form

$\frac{\mathrm{d} \chi(t)}{\mathrm{d} t}=-K_{1} \chi(t) \quad$ and $\quad \chi(t)=e^{-K_{1} t}$

\footnotetext{
* Fax: +90 2327507509 .

E-mail address: mehmetpolat@iyte.edu.tr.
}

The other widely used model is the second-order kinetic model [24-27],

$\frac{\mathrm{d} \chi(t)}{\mathrm{d} t}=-K_{2} \chi(t)^{2} \quad$ and $\quad \chi(t)=\frac{1}{1+K_{2} t}$.

If a finite amount of adsorbate remains in solution at equilibrium, Eqs. (1) and (2) need to be modified to include an adsorption capacity term, $\chi_{e}$. The Elovich equation [28], the intraparticle diffusion model $[24,27,29]$, and the modified firstorder model [30] are other examples.

The parameters of a phenomenological model are not known in advance and must be estimated from experimental data by graphing or curve-fitting procedures. They must maintain their relationships to system variables under varying conditions to be of any predictive value for scaling-up and control purposes. In cases where they do not satisfy this requirement [31], the model is modified to expand its applicability. Dividing the adsorption process into various time segments $[27,29,32]$ or redefining the rate constants as functions of the adsorbed amounts [30] are attempts of this kind.

The phenomenological model proposed in this paper is based on the interactions of the solution adsorbate with the ad- 
sorbent or surface adsorbate species. The model yields kinetic equations that associate the adsorbed amounts to the fraction of the adsorbent surface occupied. In this paper, the parameters of the model were defined for a molecular adsorbate dissolved in liquid, but they can easily be modified for other dispersed systems. One good example is the adsorption of nanoparticles onto colloidal surfaces from liquid or gas media.

The model was tested with the sodium stearate/water/ $\alpha$ alumina system for the specific reasons that (i) $\alpha$-alumina provides essentially a nonporous, homogeneous surface; (ii) the stearate molecules produce multilayers on $\alpha$-alumina [33-36]; (iii) adsorption takes place through both chemisorption (the carboxylate-surface hydroxyl bonds) [36-38] and physisorption (the stearate-stearate hydrocarbon chain bonds) $[35,36,38$, $40-42]$.

\section{Model}

For a system containing $v_{i}$ dissolved adsorbate species and a well-dispersed, homogeneous, nonporous adsorbent with $\alpha_{i}$ surface sites, disappearance of the adsorbate species from solution can be given by the following rate equation if the adsorbate-adsorbent and adsorbate-surface adsorbate affinities have finite values:

$\frac{\mathrm{d} v(t)}{\mathrm{d} t}=-k_{\mathrm{s}} v(t) \alpha(t)-k_{\mathrm{a}} v(t)\left[\alpha_{i}-\alpha(t)\right]$.

Similarly, the occupation of the adsorbent surface sites will follow the rate equation

$\frac{\mathrm{d} \alpha(t)}{\mathrm{d} t}=-k_{\mathrm{s}} v(t) \alpha(t)$.

The rate constants $k_{\mathrm{s}}$ and $k_{\mathrm{a}}$ are the probabilities that the solution adsorbate species will successfully adsorb onto the free or occupied surface sites, respectively. This definition of $k_{\mathrm{s}}$ and $k_{\mathrm{a}}$ allows unsystematic desorption.

If $v_{\mathrm{a}}$ and $\alpha_{\mathrm{a}}$ are the number concentrations of the surface adsorbate and the occupied sites on the surface, mass balance requires that $v_{\mathrm{a}}(t)=v_{i}(t)-v(t)$ and $\alpha_{\mathrm{a}}(t)=\alpha_{i}(t)-\alpha(t)$. Though $v_{\mathrm{a}}(t)$ is strictly equal to $\alpha_{\mathrm{a}}(t)$ for monolayer adsorption, the functional relation between the two is not known in the case of multilayer adsorption. Obtaining such a relation would provide important clues to the distribution of the adsorbate over the surface.

For a molecular adsorbate, $v(t)=c(t) N_{\mathrm{av}} / M_{\mathrm{w}}$ and $\alpha(t)=$ $m(t) S_{\mathrm{p}} / p$, where $c(t)$ and $m(t)$ are the mass concentrations. Defining dimensionless fractional concentrations such that $\chi(t)=c(t) / c_{i}$ and $\mu(t)=m(t) / m_{i}$, the above rate equations become

$\frac{\mathrm{d} \chi(t)}{\mathrm{d} t}=-\kappa_{\mathrm{s}} \chi(t) \mu(t)-\kappa_{\mathrm{a}} \chi(t)[1-\mu(t)]$

and

$\frac{d \mu(t)}{\mathrm{d} t}=-\lambda \kappa_{\mathrm{s}} \chi(t) \mu(t)$,

or in combined form,

$\frac{\mathrm{d} \chi(t)}{\mathrm{d} t}=\lambda \frac{\mathrm{d} \mu(t)}{\mathrm{d} t}-\kappa_{\mathrm{a}}[1-\mu(t)] \chi(t)$.
Here, $\kappa_{\mathrm{S}}$ and $\kappa_{\mathrm{a}}$ are true rate constants in reciprocal units of time, $\chi_{\mathrm{a}}=1-\chi(t)$, and $\mu_{\mathrm{a}}=1-\mu(t)$. The dimensionless term $\lambda$ is an important system parameter that is equal to the ratio of the monolayer surface area required by the initial amount of the adsorbate $\left(N_{\mathrm{av}} p c_{i} / M_{\mathrm{w}}\right)$ to the surface area provided by the adsorbent in the system $\left(S_{\mathrm{p}} m_{i}\right)$. It will be called "the Loading Factor," since it gives information on how heavily charged the adsorbent initially is with respect to the adsorbate.

For dilute adsorbate systems where $\alpha_{i} \gg v_{i}, \mu(t)$ is nearly constant and unity during adsorption. In this case, the number of interactions between the adsorbate and adsorbent will be much larger than that between the solution and surface adsorbate species. Similarly, for those systems where $\kappa_{\mathrm{S}} \approx \kappa_{\mathrm{a}}$, there is no distinction between free and occupied surface sites and the adsorbate will adsorb unhindered. For both cases, Eq. (5) can easily be shown to reduce to the first-order rate expression (Eq. (1)).

The loading factor $\lambda$ will approach unity if the initial number of adsorbate species $v_{i}$ and the initial number of surface sites $\alpha_{i}$ become comparable. For monolayer adsorption where $v_{i}-$ $v(t)=\alpha_{i}-\alpha(t)$, this would also mean $v(t)=\alpha(t)$. Hence, for $\lambda \rightarrow 1$ and $\kappa_{\mathrm{a}} \rightarrow 0, \chi(t)$ would always be equal to $\mu(t)$ during adsorption and Eq. (5) becomes analogous to the second-order rate expression (Eq. (3)) for this specific case. Thus, both the first-order and second-order rate equations are special solutions of the proposed model.

Dividing Eqs. (5) by (6) and dropping the time terms in parentheses for clarity yields

$\lambda \frac{\mathrm{d} \mu}{\mathrm{d} \chi}=1+\frac{\kappa_{\mathrm{a}}}{\kappa_{\mathrm{s}}} \frac{1-\mu}{\mu}$.

The solution of this equation for $\mu(1)=1$ is given as

$\mu=\frac{\kappa_{\mathrm{a}}}{\kappa_{\mathrm{s}}-\kappa_{\mathrm{a}}} W\left[\frac{\kappa_{\mathrm{s}}-\kappa_{\mathrm{a}}}{\kappa_{\mathrm{a}}} e^{\left(\kappa_{\mathrm{s}}-\kappa_{\mathrm{a}}\right) / \kappa_{\mathrm{a}}} e^{-\left(\kappa_{\mathrm{s}} / \kappa_{\mathrm{a}}\right) \lambda(1-\chi)}\right]$,

where the function $W(a)$ is the Lambert's $W$ or the ProductLog function such that $W\left(a e^{a}\right)=a$. Commercial mathematical software packages such as Mathematica or MathCad have $W(a)$ as a built-in function just like $\sin (a)$ or $\log (a)$. Equation (9) is an explicit relationship correlating the adsorbed amounts to the fraction of the surface occupied. It is a kinetic equation because each $\chi$ corresponds to a specific time of adsorption and provides an important tool for studying multilayer adsorption with respect to the distribution of the adsorbate on the surface both as a function of time and at equilibrium. Since $\chi$ can obtained readily from a kinetic adsorption experiment and $\lambda$ is a known design parameter, evaluation of Eq. (9) requires only the estimates of the rate constants $\kappa_{\mathrm{s}}$ and $\kappa_{\mathrm{a}}$.

The average number of adsorbed layers over the entire surface is linearly related to the solution adsorbate concentrations by

$\phi_{\mathrm{s}}=v_{\mathrm{a}}=\lambda(1-\chi)$.

However, $\phi_{\mathrm{S}}$ is only a surface-average quantity and offers no information as to how the adsorbate is distributed over the surface. When a relationship has been developed between $\chi$ and $\mu$, a more useful quantity $\phi_{\mathrm{a}}$ can be defined for the average number 

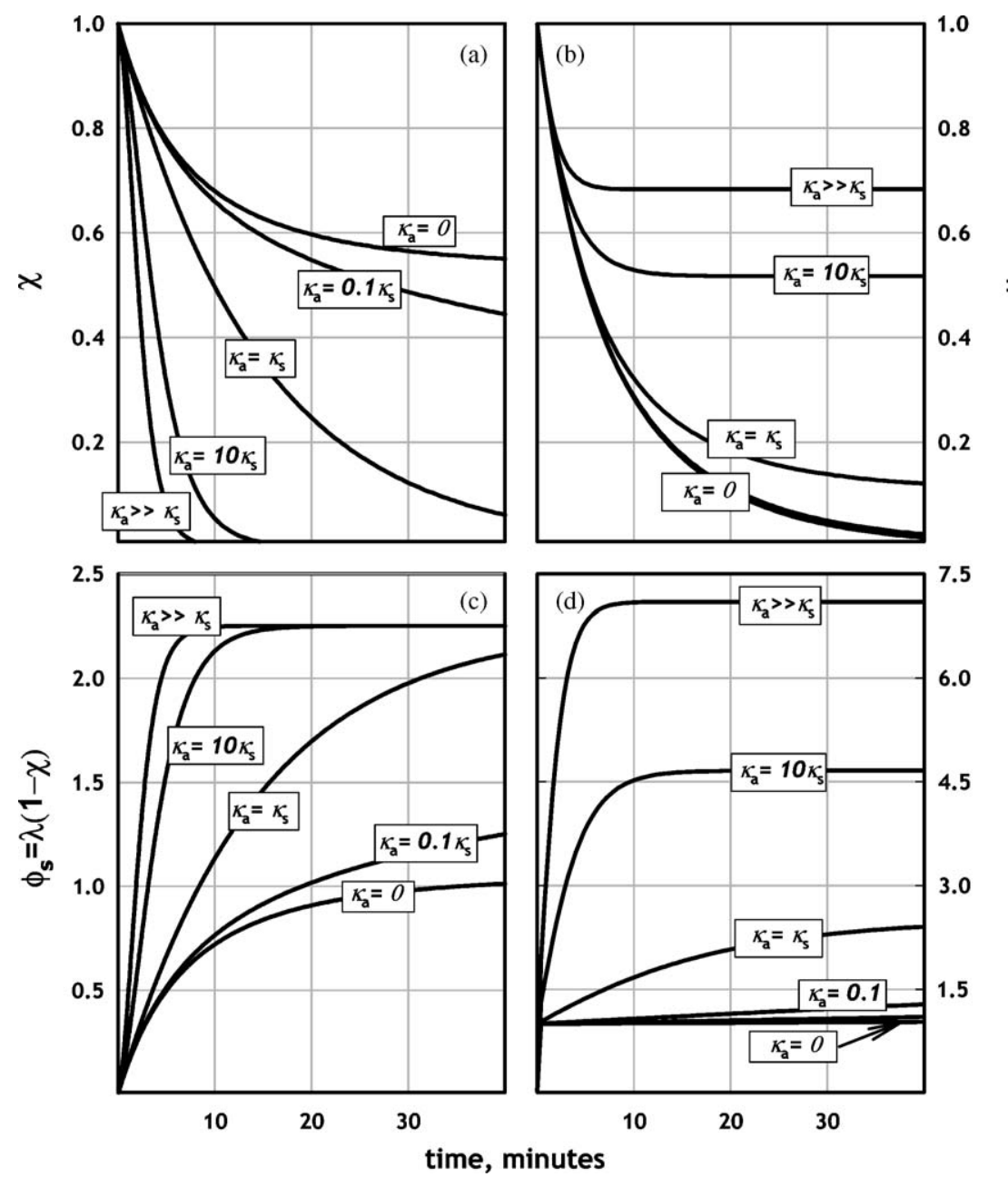

$\exists$

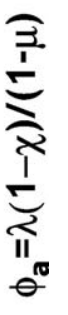

Fig. 1. Numerical solutions of Eq. (7) for different $\kappa_{\mathrm{a}} / \kappa_{\mathrm{S}}$ ratios: (a) fractional residual adsorbate concentrations, (b) fractional free surface site concentrations, (c) average number of adsorbate layers on the entire surface, (d) average number of adsorbate layers over the occupied sites alone $\left(\lambda=2.25 ; \kappa_{\mathrm{S}}=0.1 \mathrm{~min}^{-1}\right)$.

of adsorbed layers strictly over the occupied sites:

$\phi_{\mathrm{a}}=\frac{\nu_{\mathrm{a}}}{\alpha_{\mathrm{a}}}=\frac{\lambda(1-\chi)}{1-\mu}$.

Note that where as $\phi_{\mathrm{s}}$ is bound between 0 and $\lambda, \phi_{\mathrm{a}}$ is bound between 1 and any arbitrarily large number, depending on the respective values of $\chi$ and $\mu$. These quantities together provide important insights into the surface distribution of the adsorbate species. For example, a large $\phi_{\mathrm{a}}$ value for a large $\mu$ would mean that the adsorbate species are piling up on each other to form surface clusters rather than homogeneously spreading over the surface.

Some example numerical solutions of Eq. (7) are given in Fig. 1 to demonstrate how $\chi, \mu, \phi_{\mathrm{s}}$, and $\phi_{\mathrm{a}}$ vary with time for different $\kappa_{\mathrm{a}} / \kappa_{\mathrm{s}}$ ratios. In these calculations, the number of adsorbate molecules in the system is set to be 2.25 times more than the number of surface sites $(\lambda=2.25)$.

Fig. 1a shows that a large amount of the adsorbate remains unadsorbed in the case of monolayer adsorption. However, the adsorbate is completely consumed for high $\kappa_{\mathrm{a}}$ values since it can also adsorb onto the occupied sites. Despite the larger amounts of adsorbate consumed, the fraction of the free sites on the surface increases with increasing $\kappa_{\mathrm{a}} / \kappa_{\mathrm{S}}$ ratio (Fig. 1b). This means that isolated areas of multiple adsorbate layers start to form on the surface. This reasoning implies that no adsorption on the surface will take place at some high enough $\kappa_{\mathrm{a}}$ where the adsorbate species will prefer to aggregate in solution rather than attaching onto the free surface sites.

The average numbers adsorbed layers on the entire surface and on the occupied sites alone are given in Figs. 1c and 1d, respectively. For the monolayer case, $\phi_{\mathrm{s}}$ varies between 0 and 1 , whereas $\phi_{\mathrm{a}}$ is always 1 even for $\lambda$ values as high as 2.25. For increasing values of $\kappa_{\mathrm{a}}, \phi_{\mathrm{s}}$ becomes greater than 1 and always approaches the $\lambda$ value of 2.25 as adsorption proceeds. This falsely implies that the surface will always end up having an average of 2.25 homogeneous layers at extended adsorption times, irrespective of the $\kappa_{\mathrm{a}} / \kappa_{\mathrm{S}}$ ratio. However, $\phi_{\mathrm{a}}$ tends to become arbitrarily larger with increasing $\kappa_{\mathrm{a}}$. For a very high $\kappa_{\mathrm{a}} / \kappa_{\mathrm{s}}$ ratio, the adsorbate may produce a seven-layer-thick coverage on the surface while $70 \%$ of the surface is still unoccupied (see also Fig. 1b). Such vastly different pictures of the same physical situation demonstrate the merits of having knowledge of $\mu$ and $\phi_{\mathrm{a}}$ rather than $\chi$ and $\phi_{\mathrm{s}}$ alone. 

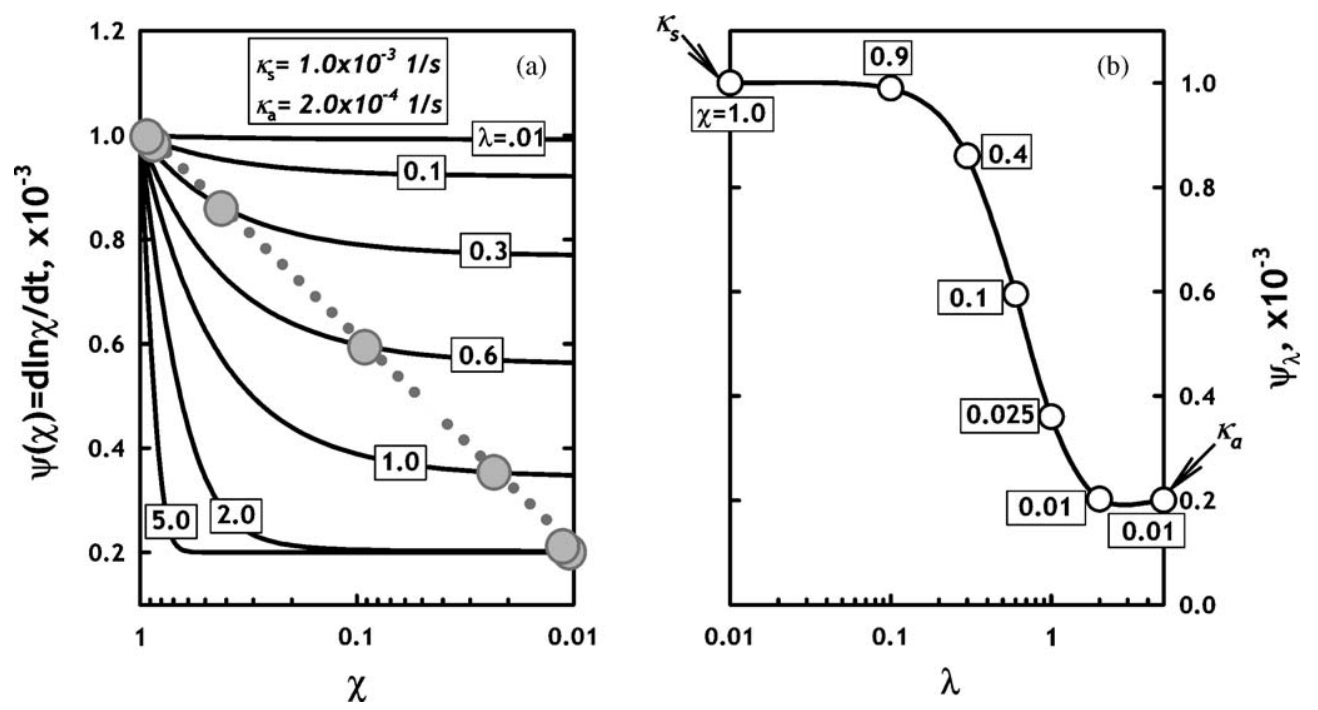

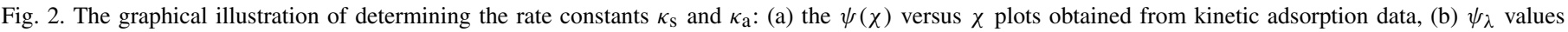
obtained from the $\psi(\chi)$ versus $\chi$ plots.

\subsection{Procedure for determination of $\kappa_{\mathrm{s}}$ and $\kappa_{\mathrm{a}}$ from kinetic adsorption data}

Replacing Eq. (9) in Eq. (5) gives

$\frac{\mathrm{d} \chi}{\mathrm{d} t}=-\psi(\chi) \chi$,

where

$\psi(\chi)=\kappa_{\mathrm{a}}\left[1+W\left(\frac{\kappa_{\mathrm{s}}-\kappa_{\mathrm{a}}}{\kappa_{\mathrm{a}}} e^{\left(\kappa_{\mathrm{s}}-\kappa_{\mathrm{a}}\right) / \kappa_{\mathrm{a}}} e^{-\left(\kappa_{\mathrm{s}} / \kappa_{\mathrm{a}}\right) \lambda(1-\chi)}\right)\right]$.

It is immediately apparent that disappearance of the adsorbate follows a dynamic first-order rate equation with a variable rate constant $\psi(\chi)$. Since $W\left(a e^{a}\right)=a$ and $W(0)=0$, Eq. (12) reduces to the first-order rate equation with true rate constants $\kappa_{\mathrm{S}}$ and $\kappa_{\mathrm{a}}$. Expressed slightly differently, it takes the forms

$-\frac{1}{\chi} \frac{\mathrm{d} \chi}{\mathrm{d} t}=\frac{\mathrm{d} \ln (\chi)}{\mathrm{d} t}=\kappa_{\mathrm{s}}, \quad$ as $\lambda \rightarrow 0$ and $\chi \rightarrow 1$,

$-\frac{1}{\chi} \frac{\mathrm{d} \chi}{\mathrm{d} t}=\frac{\mathrm{d} \ln (\chi)}{\mathrm{d} t}=\kappa_{\mathrm{a}}, \quad$ as $\lambda \rightarrow \infty$ and $\chi \rightarrow 0$.

In practical terms, Eq. (14) arises because the adsorbent surface is nearly free of any adsorbate molecules for diminishing loading factors and early adsorption times and the interactions between the adsorbate and adsorbent largely dominate the adsorption process. Similarly, Eq. (15) will result at the other extreme because the surface would be mostly occupied by the adsorbate for high loading factors and late adsorption times and the dominating interactions will be between the solution and surface adsorbate species.

Fig. 2 depicts the above paragraph in graphical form. In Fig. $2 \mathrm{a}, \psi(\chi)$ is plotted as a function of $\chi$ for different values of $\lambda$. Note that the ordinate $\psi(\chi)$ is equivalent to $\mathrm{d} \ln (\chi) / \mathrm{d} t$ (see Eqs. (12)-(14)). As predicted by Eq. (14), the $\psi(\chi)$ versus $\chi$ curves approach a constant $\kappa_{\mathrm{s}}$ value for low $\lambda$ and large $\chi$ of the upper left-hand corner and a constant $\kappa_{\mathrm{a}}$ value for high $\lambda$ and low $\chi$ of the lower right-hand corner. This feature of the model can be exploited to determine $\kappa_{\mathrm{S}}$ and $\kappa_{\mathrm{a}}$ accurately. It is based on determining a characteristic $\psi_{\lambda}$ value for each $\psi(\chi)$ versus $\chi$ plot given in Fig. $2 \mathrm{a}$ and creating a $\psi_{\lambda}$ versus $\lambda$ plot. The $\psi_{\lambda}$ values should be chosen so that the selection moves progressively from $\kappa_{\mathrm{s}}$-dominated regions (large $\chi$-small $\lambda$ ) to $\kappa_{\mathrm{a}}$-dominated regions (small $\chi$-large $\lambda$ ) as $\lambda$ increases. This would mean moving diagonally from the upper left corner to the lower right corner in Fig. 2a as shown with a dotted diagonal line for illustrative purposes. Such diagonal selection of the $\psi(\chi)$ values also minimizes any instrumental resolution difficulties in the analysis of the adsorbate at low $\lambda$ values by sliding the selection to higher $\chi$ values progressively. The characteristic $\psi_{\lambda}$ values that fall on the dotted dashed line (shown with filled circles in Fig. 2a) are plotted as a function of $\lambda$ in Fig. 2b. It can be seen that the $\psi_{\lambda}$ plot smoothly approaches $\kappa_{\mathrm{S}}$ for $\lambda \rightarrow 0$ and $\chi \rightarrow 1$ and $\kappa_{\mathrm{a}}$ for $\lambda \rightarrow \infty$ and $\chi \rightarrow 0$.

Usually, a kinetic adsorption experiment is carried out using incremental time intervals. Expressing Eqs. (12) and (13) in incremental terms would be helpful in clarifying the meaning of the various terms in these equations and how they are determined in an adsorption experiment:

$$
\begin{aligned}
\frac{1}{\bar{\chi}} \frac{\Delta \chi}{\Delta t} & =\psi(\bar{\chi}) \\
& =-\kappa_{\mathrm{a}}\left[1+W\left(\frac{\kappa_{\mathrm{s}}-\kappa_{\mathrm{a}}}{\kappa_{\mathrm{a}}} e^{\left(\kappa_{\mathrm{s}}-\kappa_{\mathrm{a}}\right) / \kappa_{\mathrm{a}}} e^{-\left(\kappa_{\mathrm{s}} / \kappa_{\mathrm{a}}\right) \lambda(1-\bar{\chi})}\right)\right] .
\end{aligned}
$$

Here, $\Delta \chi$ is simply the fractional amount adsorbed within time interval $\Delta t$ and is directly determined in an adsorption experiment. The term $\bar{\chi}$ is the average residual adsorbate concentration within the same time interval and can be calculated readily from knowledge of $\chi_{i}$ and $\Delta \chi$. Therefore, a plot of $\psi(\bar{\chi})$ versus $\bar{\chi}$ can easily be obtained from a kinetic adsorption experiment to generate Figs. $2 \mathrm{a}$ and $2 \mathrm{~b}$. Since this procedure requires multiple kinetic adsorption experiments at different loading factors, the estimated values of the rate constants should be reliable. 
By definition, $\kappa_{\mathrm{S}}$ and $\kappa_{\mathrm{a}}$ are true rate constants and should not change with changes in the loading factor $\lambda$. However, for a given volume of solution, a $\lambda$ value of 0.1 may mean either $10 \mathrm{mg}$ adsorbate per $0.1 \mathrm{~g}$ solids or $1000 \mathrm{mg}$ adsorbate per $10 \mathrm{~g}$ solids. Since the solution is much more dilute with respect to the adsorbate-adsorbent species in the former case compared to the latter, the probability of the adsorbate-adsorbent encounters and, therefore, the values of $\kappa_{\mathrm{s}}$ and $\kappa_{\mathrm{a}}$ will be different. Therefore, the adsorption tests should be carried out under constant adsorbent mass concentration.

\subsection{Approximate analytical solutions for determination of $\kappa_{\mathrm{S}}$ and $\kappa_{\mathrm{d}}$ by curve fitting}

Analytical solutions of Eq. (7) can be obtained for some widely encountered special cases. The rate constants $\kappa_{\mathrm{S}}$ and $\kappa_{\mathrm{a}}$ can then be estimated directly by curve fitting the analytical equations to kinetic adsorption data.

\subsubsection{Monolayer adsorption $\left(\kappa_{\mathrm{a}}=0\right)$ case}

If the affinity between the solution and surface adsorbate species is diminishingly low, the adsorbate will adsorb as a monolayer. In this case, Eq. (5) reduces to $\frac{\mathrm{d} \chi(t)}{\mathrm{d} t}=-\kappa_{\mathrm{s}} \chi \mu$. Since $\alpha_{\mathrm{a}}(t)$ is always equal to $v_{\mathrm{a}}(t)$ for monolayer adsorption, it can be shown in fractional terms that $\mu=1-\lambda(1-\chi)$. Therefore, Eq. (5) becomes

$$
\frac{\mathrm{d} \chi}{\mathrm{d} t}=-\kappa_{\mathrm{s}} \chi[1-\lambda(1-\chi)] .
$$

The solution for $\chi(0)=1$ is equal to

$\chi=\frac{\lambda-1}{\lambda-e^{-\kappa_{\mathrm{s}}(\lambda-1) t}}$.

Equation (18) reduces to the first-order and second-order rate equations at the limits $\lambda \rightarrow 0$ and $\lambda \rightarrow 1$, respectively. For $0<\lambda \leqslant 1$, the adsorbate would completely adsorbs onto the adsorbent given enough time for adsorption. For $\lambda>1$, some adsorbate remains unadsorbed irrespective of the time of adsorption. This amount can be seen to be equal to $(\lambda-1) / \lambda$ from Eq. (18) as $t \rightarrow \infty$ and is equivalent to the adsorption capacity term $\chi_{\mathrm{e}}$ mentioned earlier.

\subsubsection{Stronger adsorbate-adsorbent affinities $\left(\kappa_{\mathrm{s}} \geqslant \kappa_{\mathrm{a}}\right)$ case}

If the adsorbate-adsorbent affinity is no less than the adsorbate-surface adsorbate affinity, an approximate analytical solution of Eq. (7) can be obtained as

$\chi(t)=\frac{(\lambda-1) e^{-\kappa_{\mathrm{a}} t}}{\lambda-e^{-\left(\kappa_{\mathrm{s}}-\kappa_{\mathrm{a}}\right)(\lambda-1) t}}$.

Such a condition is satisfied for most adsorption systems. Mathematically, Eq. (19) becomes analogous to the first-order equation (Eq. (1)) for $\lambda \rightarrow 0$, to the second-order equation (Eq. (2)) for $\lambda \rightarrow 1$ and $\kappa_{\mathrm{a}} \rightarrow 0$, and to the monolayer adsorption equation (Eq. (18)) for $\kappa_{\mathrm{a}} \rightarrow 0$.

\section{Experimental: testing of the model using an $\alpha$-alumina/sodium stearate system}

\subsection{Material}

A commercial high-purity spherical $\alpha-\mathrm{Al}_{2} \mathrm{O}_{3}$ powder (99.99\% purity; AKP-53 from Sumitomo Chemicals, Japan) was used in this study (Fig. 3). The volume and number mean diameters were $0.290 \mu \mathrm{m}$ and $0.13 \mu \mathrm{m}$. A simple calculation assuming spherical, nonporous particles gives a surface area of about $12 \mathrm{~m}^{2} / \mathrm{g}$. This value agrees well with the measured BET surface area of $12.3 \mathrm{~m}^{2} / \mathrm{g}$ provided by the manufacturer, indicating the nonporous structure of the powder.

Sodium stearate (99+ purity; Aldrich, USA) was used without further purification. Its $\mathrm{CMC}$ was measured to be $1.7 \times 10^{-3} \mathrm{M}(0.55 \mathrm{~g} / \mathrm{L})$ at $25^{\circ} \mathrm{C}$ and $\mathrm{pH} 9.2$ by a Kruss K10ST digital tensiometer employing the Wilhelmy plate method. This is in close agreement with the literature value of $1.8 \times 10^{-3} \mathrm{M}$ [43]. The surface tension-log concentration data resulted in a well-defined straight line below the CMC and a sharp turn very close to CMC, an indication that transition to micellar forms was in a narrow concentration range and monomeric forms were dominant below CMC. The highest initial stearate concentration used in the experiments was always well below the CMC to ensure an abundance of monomers in solution. Double distilled water that was passed through a Barnstead EasyPure UV compact ultrapure water system (1.8 M $\Omega$ ) was used throughout the work.

The values of $m_{i}, c_{i}, S_{\mathrm{p}}$, and $p$ had to be known to the loading factor $\lambda$. The initial solids concentration $m_{i}$ was kept constant at $20 \mathrm{~g} / \mathrm{L}$ in all test while the initial adsorbate concentration $c_{i}$ was varied. The solids concentration of $20 \mathrm{~g} / \mathrm{L}$ was selected to provide enough surface area for the stearate molecules at the stearate concentrations used so that the $\lambda$ values would cover a range between about 0.1 and 2 . Stearic acid was reported to have a parking area between 0.2 [34,43-45] and $0.7 \mathrm{~nm}^{2}$ [46-49] depending on the orientation of the molecule on the surface. A more recent work reported a parking area of $0.47 \mathrm{~nm}^{2}$ [36] where the stearate molecule was proposed to adsorb binuclearly onto two aluminum atoms through the carboxylate group. Using the latest value as the parking area, $\lambda$ for

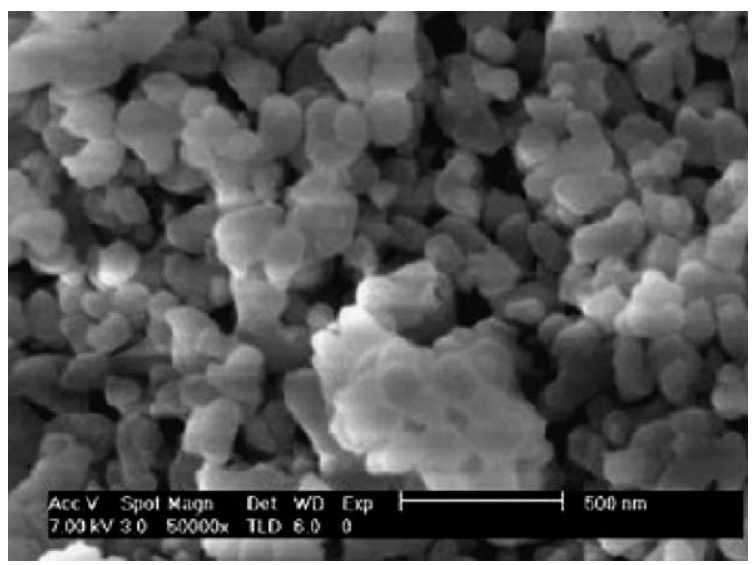

Fig. 3. The SEM picture of the $\alpha-\mathrm{Al}_{2} \mathrm{O}_{3}$ powder used in the study. 
the experimental system comes out to be $3.75 \times 10^{-3} c_{i}$, where $c_{i}$ is the solution stearate concentration in $\mathrm{mg} / \mathrm{L}$, which was varied between 30 and $500 \mathrm{mg} / \mathrm{L}$ in this study, giving a $\lambda$ range of 0.113 to 1.876 .

\subsection{Method}

Adsorption experiments were carried out in 250-ml polyethylene bottles under vigorous shaking. The $\mathrm{pH}$ was adjusted to 9.2 to operate at the pzc of the powder where maximum stearate adsorption was observed [39-42] and to prevent precipitation of sodium stearate. The solid concentration in each bottle was adjusted to $20 \mathrm{~g} / \mathrm{L}$ in all experiments. The bottles were ultrasonified for $30 \mathrm{~min}$ to break any agglomerates and were transferred to a shaker. A proper aliquot of the adsorbate was added to each bottle from a stock solution to obtain the desired $c_{i}$ and $\lambda$ values. The moment of stearate addition was taken as the zero time of adsorption and the shaking was initiated. The temperature was kept constant at $20^{\circ} \mathrm{C}$ during all the adsorption tests. The sampling times were $2,4,6,8,10,15$, $20,25,30,35,40,45,50,60$, and 120 min since majority of the adsorbate was consumed within an hour. Each bottle was used to obtain only a single sample to minimize accumulation of experimental error. The Millipore Millex sterile syringe filters with average pore diameter $0.1 \mu \mathrm{m}$ were used to acquire the supernatant samples for analysis in a Shimadzu GC-2010 gas chromatograph-Py-2020iD pyrolyzer system using a capillary column and an FID detector.

\section{Results}

The results of a series of kinetic adsorption tests carried out at different loading factors $(\sim 0.1<\lambda<2)$ are presented in Fig. 4 as symbols. The test at $\lambda=0.938$, which was repeated three times, showed good reproducibility (see the inset graph figure). The solid lines in the figure were drawn for datasmoothing purposes only, using an eight-parameter exponential decay function $f(t)=\sum_{i=1}^{4} p_{2 i-1} e^{-p_{2 i} t}$.

Comparison of the data with the monolayer expression (Eq. (18)) is presented in Fig. 5 as solid lines. The experimental data and Eq. (18) agreed quite well for the low $\lambda$ values of 0.113 and 0.375 where the $\kappa_{\mathrm{S}}$ values estimated by Eq. (18) were both around $0.153 \mathrm{~min}^{-1}$. This was most probably due to the fact that the stearate-alumina interactions dominated the adsorption process at low $\lambda$ values where the initial concentration of the alumina sites was much higher than that of the stearate species. However, Eq. (18) failed to represent the data for higher $\lambda$ values. The fact that the monolayer equation failed for high $\lambda$ values was an indication of multilayer adsorption.

The $\psi(\chi)$ versus $\chi$ plots that were calculated using the smoothed lines in Fig. 4 by Eq. (16) are given in Fig. 6a for each $\lambda$. It is immediately apparent that all the plots converge towards the same $\psi(\chi)$ for all $\lambda$. This point, which corresponds to $0.15 \mathrm{~min}^{-1}$, is the rate constant $\kappa_{\mathrm{S}}$ for the system. Note that this value is very close to the $\kappa_{\mathrm{S}}$ value predicted by the monolayer equation in Fig. 5 at lower $\lambda$ values. The $\psi_{\lambda}$ values obtained from the $\psi(\chi)$ versus $\chi$ plots are plotted in Fig. $6 \mathrm{~b}$ as a function of $\lambda$. The $\psi_{\lambda}$ curve in the figure gradually approaches two

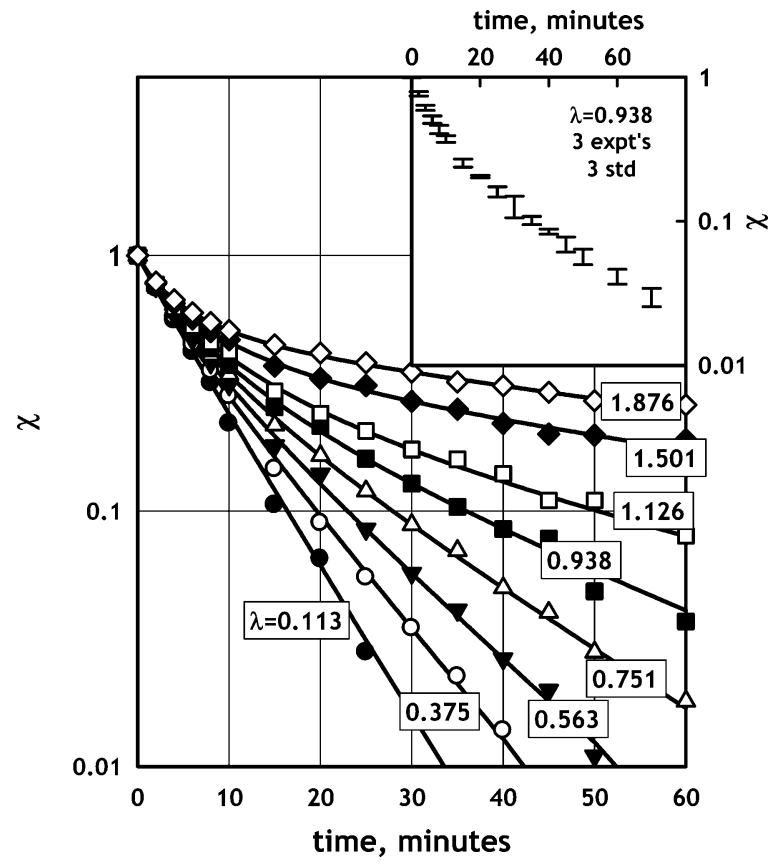

Fig. 4. Residual stearate concentrations as a function of time (symbols) for the $\alpha$-alumina/water/sodium stearate system at various $\lambda$. (The solid lines were drawn for data smoothing purposes only. The inset figure shows the three standard-deviation spread for the three repeat adsorption tests at $\lambda=0.938$.)

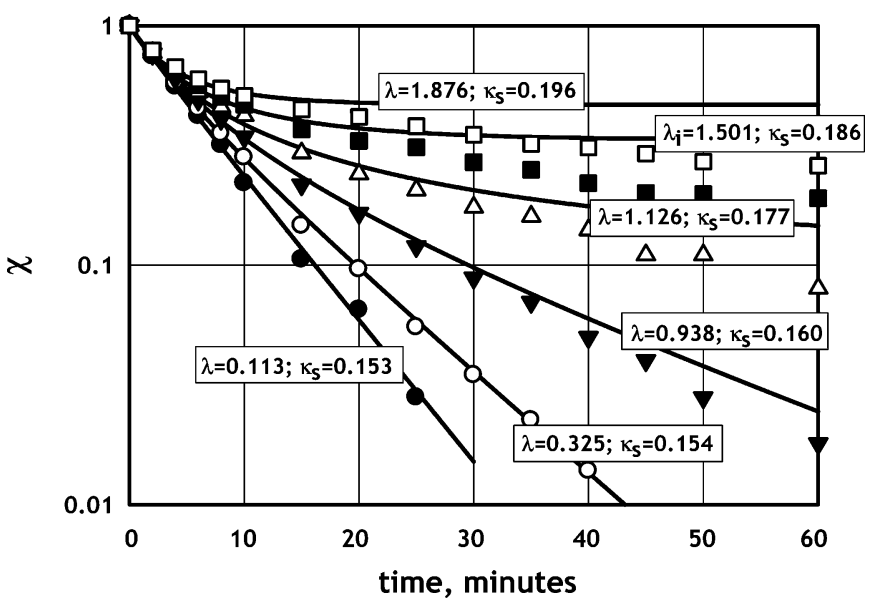

Fig. 5. Comparison of the monolayer expression (Eq. (18)—solid lines) with the experimental data from Fig. 4 (symbols).

limiting values $\lambda \rightarrow 0$ and $\lambda \rightarrow \infty$. These values are 0.150 and $0.011 \mathrm{~min}^{-1}$ and represent the magnitudes of the true rate constants $\kappa_{\mathrm{S}}$ and $\kappa_{\mathrm{a}}$ for the alumina-stearate system. The value of $\kappa_{\mathrm{a}}$ is much smaller than $\kappa_{\mathrm{s}}$, implying that the stearate-alumina interactions are much stronger that the interactions between the solution and surface stearate molecules (the stearate molecules adsorbed on the surface). This is in agreement with the literature which have suggested that the stearate adsorption is multilayer in nature and that the initial layer adsorbs strongly by chemical bonding followed by additional weakly bonded layers [36-42].

The fraction of the occupied sites $\left(\mu_{\mathrm{a}}\right)$ and the thickness of the adsorbed layers on occupied sites $\left(\phi_{\mathrm{a}}\right)$ calculated for the estimated values of $\kappa_{\mathrm{a}}$ and $\kappa_{\mathrm{S}}$ are presented in Fig. 7 for selected $\lambda$. Figs. 7a-7c, which correspond to loading factors below 1, show 

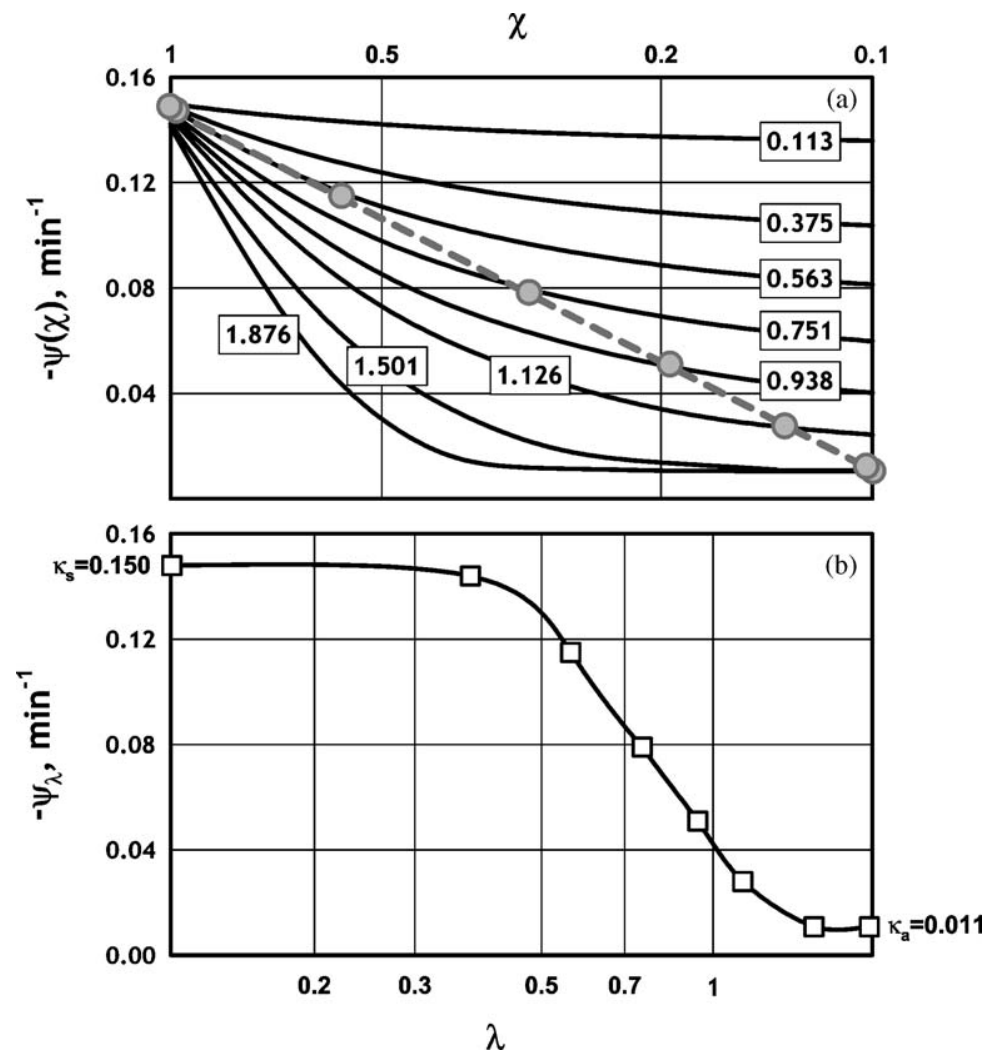

Fig. 6. Estimation of the rate constants $\kappa_{\mathrm{a}}$ and $\kappa_{\mathrm{S}}$ for the $\alpha$-alumina/water/sodium stearate system: (a) $\psi(\chi)$ versus $\chi$ plots obtained from experimental data in Fig. 4 , (b) $\psi_{\lambda}$ values obtained from the $\psi(\chi)$ versus $\chi$ plots.

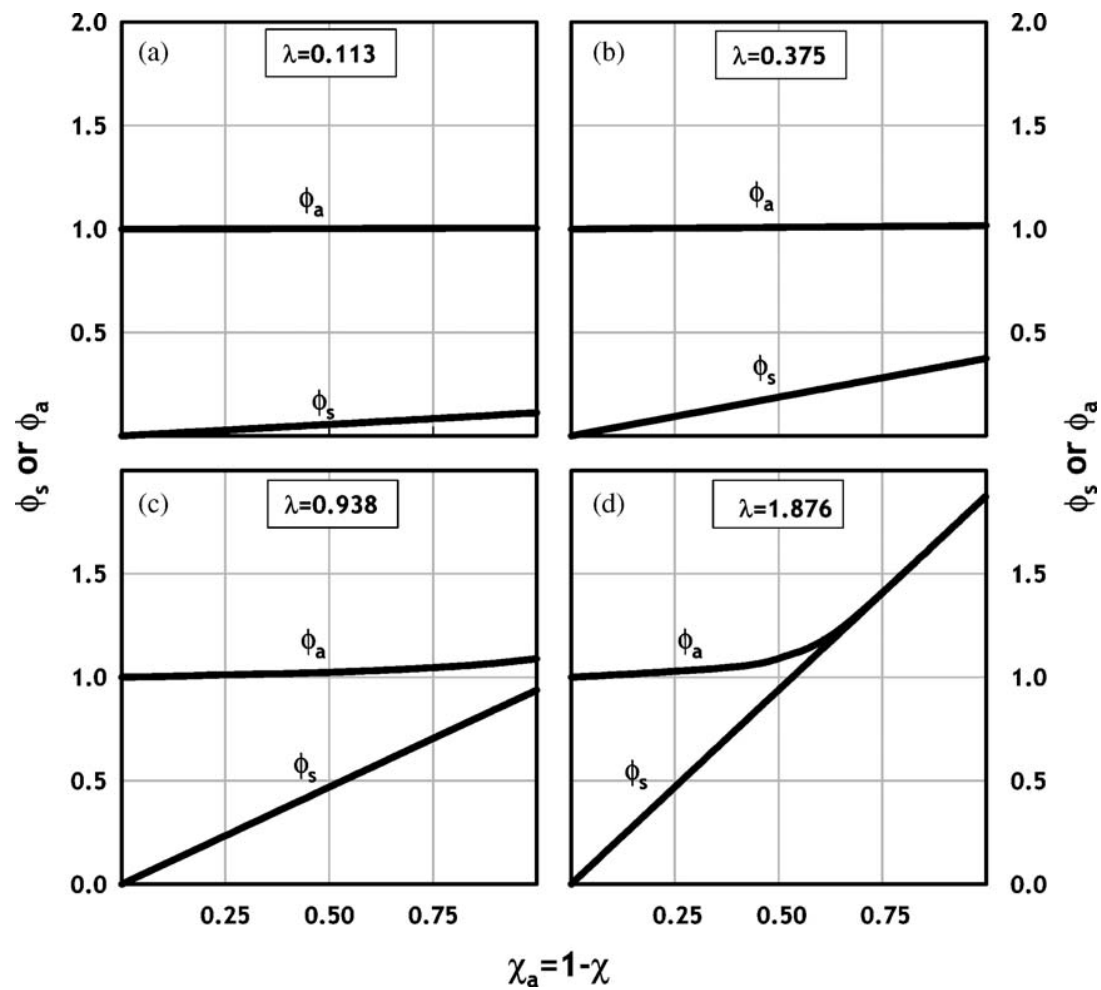

Fig. 7. The average number of adsorbed stearate layers on the entire alumina surface $\left(\phi_{\mathrm{S}}\right)$ and on the occupied alumina sites alone $\left(\phi_{\mathrm{a}}\right)$ as a function of $\chi_{\mathrm{a}}$ for selected $\lambda$. 


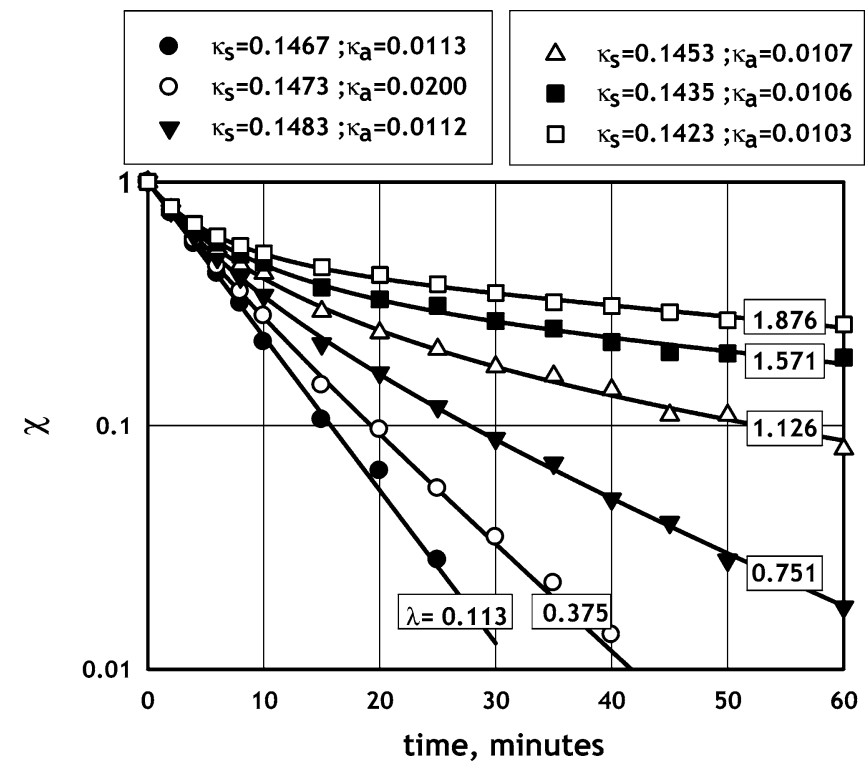

Fig. 8. Comparison of the analytical $\kappa_{\mathrm{S}} \geqslant \kappa_{\mathrm{a}}$ expression (Eq. (19)—solid lines) with the experimental data from Fig. 4 (symbols). (The parameter values in the inset boxes are the best-fit estimates of the curve fitting procedure.)

that $\phi_{\mathrm{a}}$ never exceeds 1.0. The fact that it stays very close to 1 at all times, even at $\lambda=0.938$, suggests strongly that the stearate species prefer to adsorb onto the alumina surface rather than creating a secondary layer. Fig. $7 d$ shows that the $\phi_{\mathrm{a}}$ value at $\lambda=1.876$ is well below 2 , indicating that the stearate species do not produce a third adsorbed layer within the concentration range studied, which was below the CMC. This agrees with the reported behavior that the stearate forms surface hemimicelles of double molecules by hydrophobic bonding below the CMC. All the plots in Fig. 7 show that $\phi_{\mathrm{s}}$ and $\phi_{\mathrm{a}}$ curves approach each other as $\chi$ and $\lambda$ becomes larger. This indicates a homogeneous distribution of the adsorbate where the stearate species essentially complete a monolayer before starting to adsorb as a second layer.

A comparison of Eq. (19) with the experimental data is presented in Fig. 8 as solid lines. The values of $\kappa_{\mathrm{a}}$ and $\kappa_{\mathrm{S}}$ estimated by Eq. (19) are given in the boxes above the graph for each $\lambda$. The figure demonstrates not only that Eq. (19), which is valid for $\kappa_{\mathrm{s}} \geqslant \kappa_{\mathrm{a}}$, represents the data extremely well for the entire concentration range, but also that the predicted rate parameters are quite consistent for all $\lambda$ values and very close to those determined in Fig. 6 by the graphical procedure.

\section{Conclusions}

A phenomenological kinetic multilayer adsorption model that is based on interactions of the solution adsorbate species with the adsorbent or the surface adsorbate species is proposed in this paper. The model assumes that the adsorption system consists of a well-dispersed, nonporous, homogeneous adsorbent and a molecular adsorbate below the aggregation concentrations. The widely used first- and second-order models are special solutions of the model.

One of the main parameters of the model is a dimensionless constant $\lambda$, which was called "the loading factor" in this pa- per. It is the ratio of the monolayer surface area required by the adsorbate to the surface area of the adsorbent. The other parameters are true rate constants related to the adsorbate-adsorbent $\left(\kappa_{\mathrm{S}}\right)$ and adsorbate-surface adsorbate affinities $\left(\kappa_{\mathrm{a}}\right)$.

While the loading factor is known for well-defined systems, the rate constants must be determined from kinetic adsorption data. A robust general graphical procedure was outlined for determination of the rate constants from multiple adsorption data carried out at different $\lambda$ and should be statistically reliable. Analytical expressions for the monolayer adsorption and stronger adsorbate-adsorbent interactions were also provided to estimate the model parameters by curve fitting.

The model yields analytical equations that explicitly associate the adsorbed amounts to the fraction of the surface covered. These equations should be important tools for studying the distribution of the adsorbate on the adsorbent in multilayer adsorption.

The model represented the adsorption of sodium stearate onto $\alpha$-alumina from water extremely well. The rate constants estimated by the model agreed with the reported behavior of the stearate/water $\alpha$-alumina system. The rate parameter $\kappa_{\mathrm{s}}$ was an order of magnitude larger than $\kappa_{\mathrm{a}}$, suggesting that the stearatealumina were much stronger than the stearate-surface stearate interactions. From the analysis of the fractional surface coverage it was deduced that the stearate molecules were distributed homogeneously on the alumina surface, essentially adsorbing as a monolayer before proceeding to the second layer.

\section{Appendix A. Nomenclature}

$c_{i} \quad$ initial adsorbate concentration $[\mathrm{g} / \mathrm{L}]$

$c(t) \quad$ concentration of adsorbate in solution at time $t[\mathrm{~g} / \mathrm{L}]$

$k_{\mathrm{S}} \quad$ the rate constant related to the probability that encounter of a solution adsorbate with adsorbent produces adsorption [L/s]

$k_{\mathrm{a}} \quad$ the rate constant related to the probability that encounter of a solution adsorbate with a surface adsorbate produces adsorption $[\mathrm{L} / \mathrm{s}]$

$K_{1} \quad$ rate constant for the first-order model [1/s]

$K_{2}$ rate constant for the second-order model [1/s]

$m_{i} \quad$ initial adsorbent mass concentration $[\mathrm{g} / \mathrm{L}]$

$m(t)$ mass concentration of the free adsorbent surface at time $t[\mathrm{~g} / \mathrm{L}] ; v_{\mathrm{a}}(t)=v_{i}-v(t)$

$M_{\mathrm{W}} \quad$ molecular weight of the adsorbate $[\mathrm{g} / \mathrm{mol}]$

$N_{\text {av }} \quad$ Avogadro's number, $6.02 \times 10^{23}[$ molecule $/ \mathrm{mol}]$

$p \quad$ parking area of the adsorbate molecule $\left[\mathrm{m}^{2} /\right.$ molecule $]$

$S_{\mathrm{p}} \quad$ specific surface area of the adsorbent $\left[\mathrm{m}^{2} / \mathrm{g}\right]$

$\alpha_{i} \quad$ initial number concentration of adsorbent surface sites $[1 / \mathrm{L}]$

$\alpha(t) \quad$ number concentration of free adsorbent surface sites at time $t[1 / \mathrm{L}]$

$\alpha_{\mathrm{a}}(t) \quad$ number concentration of occupied adsorbent surface sites at time $t[1 / \mathrm{L}]$;

$\kappa_{\mathrm{S}} \quad$ reduced form of the rate constant $k_{\mathrm{S}}[1 / \mathrm{s}] ; \kappa_{\mathrm{S}}=$ $k_{\mathrm{s}} S_{\mathrm{p}} m_{i} / p$

$\kappa_{\mathrm{a}} \quad$ reduced form of the rate constant $k_{\mathrm{a}}[1 / \mathrm{s}] ; \kappa_{\mathrm{a}}=$ $k_{\mathrm{a}} S_{\mathrm{p}} m_{i} / p$ 
$\lambda$

the dimensionless loading factor that gives the ratio of the monolayer surface area required by the initial adsorbate concentration to the surface area provided by the adsorbent; $\lambda=\left(N_{\mathrm{av}} p c_{i} / M_{\mathrm{w}}\right) /\left(S_{\mathrm{p}} m_{i}\right)$

$\mu(t)$ or $\mu$ fractional concentration of free adsorbent surface at time $t[\mathrm{~g} / \mathrm{g}] ; m(t) / m_{i}$

$\mu_{i} \quad$ initial fractional concentration of adsorbent $[\mathrm{g} / \mathrm{g}]$; $\mu_{i}=1$

$\mu_{\mathrm{a}}(t)$ or $\mu_{\mathrm{a}}$ fractional concentration of occupied adsorbent at time $t[\mathrm{~g} / \mathrm{g}] ; \mu_{\mathrm{a}}(t)=1-\mu$

$\nu_{i} \quad$ initial number concentration of adsorbate species in solution $[1 / \mathrm{L}]$

$v(t) \quad$ number concentration of solution adsorbate species at time $t[1 / \mathrm{L}]$

$v_{\mathrm{a}}(t) \quad$ number concentration of adsorbate species adsorbed on the surface at time $t[1 / \mathrm{L}] ; v_{\mathrm{a}}(t)=v_{i}-v(t)$

$\phi_{\mathrm{S}}(t) \quad$ average number of adsorbed layers over entire adsorbent surface; $\phi_{\mathrm{s}}=\lambda[1-\chi]$

$\phi_{\mathrm{a}}(t) \quad$ average number of adsorbed layers over occupied surface alone; $\phi_{\mathrm{a}}=\lambda(1-\chi) /(1-\mu)$

$\chi(t)$ or $\chi$ fractional solution adsorbate concentration at time $t$ [g/g]; $c(t) / c_{i}$

$\chi_{i} \quad$ initial fractional adsorbate concentration $[\mathrm{g} / \mathrm{g}] ; \chi_{i}=1$

$\chi_{\mathrm{a}}(t)$ or $\chi_{\mathrm{a}}$ fractional adsorbed adsorbate concentration at time $t[\mathrm{~g} / \mathrm{g}] ; \chi_{\mathrm{a}}=1-\chi$

$\chi_{\mathrm{e}} \quad$ adsorption capacity $[\mathrm{g} / \mathrm{g}]$

\section{References}

[1] R.G. Lee, T.W. Weber, Can. J. Chem. Eng. 47 (1969) 54.

[2] X. Yang, B. Al-Duri, Chem. Eng. J. 83 (2001) 15.

[3] X. Yang, S.R. Otto, B. Al-Duri, Chem. Eng. J. 94 (2003) 199.

[4] S. Lagergren, Handlingar 24 (1898) 1.

[5] H.C. Trivedi, V.M. Patel, R.D. Patel, Eur. Polym. J. 9 (1973) 525.

[6] D.B. Singh, G. Prasad, D.C. Rupainwar, V.N. Singh, Water Air Soil Pollut. 42 (1988) 373.

[7] G.S. Gupta, G. Prasad, V.N. Singh, Water Res. 24 (1990) 45.

[8] Y.C. Sharma, G.S. Gupta, G. Prasad, D.C. Rupainwar, Water Air Soil Pollut. 49 (1990) 69.

[9] Y.C. Sharma, G. Prasad, D.C. Rupainwar, J. Environ. Stud. 37 (1991) 183.

[10] C. Namasivayam, N. Kanchana, Chemosphere 25 (1992) 1691.

[11] M.M. Bhutani, R. Kumari, J. Radioanal. Nucl. Chem. 180 (1994) 145.

[12] S.P. Mishra, V.K. Singh, Radiochim. Acta 68 (1995) 251.

[13] K.S. Low, C. Lee, K.K. Tan, Bioresour. Technol. 52 (1995) 79.
[14] C.K. Lee, K.S. Low, K.L. Kek, Bioresour. Technol. 54 (1995) 183.

[15] S.A. Wasay, Md.J. Haron, A. Uchiumi, S.S. Tokunaga, Water Res. 30 (1996) 1143.

[16] S. P Mishra, V.K. Singh, D. Tiwari, Appl. Radiat. Isot. 47 (1996) 15.

[17] F.F.O. Orumwense, J. Chem. Technol. Biotechnol. 65 (1996) 363.

[18] D.B. Singh, G. Prasad, D.C. Rupainwar, Colloids Surf. A Physicochem. Eng. Aspects 111 (1996) 49.

[19] A.K. Mittal, S.K. Gupta, Water Sci. Technol. 34 (1996) 81.

[20] S.A. Wasay, Md.J. Haron, S. Tokunaga, Water Environ. Res. 68 (1996) 295.

[21] C. Namasivayam, N. Muniasamy, K. Gayatri, M.K. Rani, M.K. Ranganathan, Bioresour. Technol. 57 (1996) 37.

[22] C.K. Lee, K.S. Low, S.W. Chow, Environ. Technol. 17 (1996) 1023.

[23] C. Namasivayam, D.J.S.E. Arasi, Chemosphere 34 (1997) 401.

[24] F.C. Wu, R.L. Tseng, R.S. Juang, Water Res. 35 (2001) 613.

[25] C.Y. Chang, W.T. Tsai, C.H. Ing, C.F. Chang, J. Colloid Interface Sci. 260 (2003) 273.

[26] A.S. Özcan, B. Erdem, A. Özcan, J. Colloid Interface Sci. 280 (2004) 44.

[27] M.Y. Chang, R.S. Juang, J. Colloid Interface Sci. 278 (2004) 18.

[28] M.J.D. Low, Chem. Rev. 60 (1960) 267.

[29] S.J. Allen, G. McKay, K.Y.H. Khader, Environ. Pollut. 56 (1989) 39.

[30] X. Yang, B. Al-Duri, J. Colloid Interface Sci. 287 (2005) 25.

[31] S.C. Biswas, D.K. Chattoraj, J. Colloid Interface Sci. 205 (1998) 12.

[32] S.Y. Quek, Adsorption of Heavy Metal from Aqueous Solution by Natural Low-Cost Materials, Ph.D. Thesis, The University of Birmingham, 1998.

[33] M. Hasegawa, M.J.D. Low, J. Colloid Interface Sci. 30 (1969) 378.

[34] D.I. Husbands, W. Tallis, J.C.R. Waldsax, C.R. Woodings, M.J. JayCock, Powder Technol. 5 (1971/1972) 31.

[35] J.J. Zullig, J.W. Morse, Geochim. Cosmochim. Acta 52 (1988) 1667.

[36] H.A. Capelle, L.G. Britcher, G.E. Morris, J. Colloid Interface Sci. 268 (2003) 293.

[37] M.B. McBride, J. Colloid Interface Sci. 76 (1980) 393.

[38] P. Fenter, N.C. Sturchio, Geochim. Cosmochim. Acta 63 (1999) 3145.

[39] M.G. Song, J.Y. Kim, J.D. Kim, Colloids Surf. A Physicochem. Eng. Aspects 229 (2003) 75.

[40] M.A. Rahman, S.K. Basu, A. Roychoudhury, A.K. Ghosh, J. Colloid Interface Sci. 85 (1982).

[41] A.P. Oliveira, M.L. Torem, Colloids Surf. A Physicochem. Eng. Aspects 110 (1996) 75.

[42] T.V.V. Kumar, S. Prabhakar, G. Bhaskar Raju, J. Colloid Interface Sci. 247 (2002) 275.

[43] D. Beneventi, B. Carre, A. Gandini, Colloids Surf. A Physicochem. Eng. Aspects 189 (2001) 65.

[44] W.D. Harkins, D.M. Grans, J. Am. Chem. Soc. 53 (1931) 2804.

[45] A.S. Russel, C.N. Cochran, Ind. Eng. Chem. 42 (1950) 1332.

[46] A.J. Groszek, Chem. Ind. (London) (1966) 1754.

[47] T. Allen, R.M. Patel, J. Appl. Chem. 20 (1970) 165.

[48] M.A. Rahman, A.K. Ghosh, Appl. Surf. Sci. 5 (1980) 332.

[49] M.A. Rahman, Bangladesh J. Sci. Ind. Res. 12 (1977) 151. 